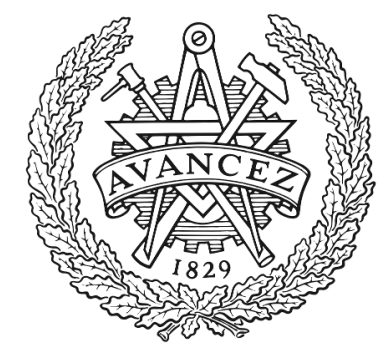

CHALMERS

UNIVERSITY OF TECHNOLOGY

\title{
Investigation on Numerical Prediction of Propeller Induced Hull Pressure Pulses
}

Downloaded from: https://research.chalmers.se, 2023-04-26 07:07 UTC

Citation for the original published paper (version of record):

Ge, M., Bensow, R., Svennberg, U. (2018). Investigation on Numerical Prediction of Propeller Induced Hull Pressure Pulses. Proceedings of the 10th International Symposium on Cavitation (CAV2018). http://dx.doi.org/10.1115/1.861851_ch123

N.B. When citing this work, cite the original published paper. 


\title{
Investigation on Numerical Prediction of Propeller Induced Hull Pressure Pulses
}

\author{
Muye $\mathrm{Ge}^{1}$; Rickard E. Bensow ${ }^{1}$; Urban Svennberg ${ }^{2}$; \\ ${ }^{1}$ Chalmers University of Technology, Sweden; ${ }^{2}$ Rolls Royce Hydrodynamic Research Center, Sweden
}

\begin{abstract}
Simulation of a cavitating propeller in behind conditions and analysis of induced hull pressure fluctuations are presented. All the simulations were performed using RANS method in the commercial package Star-CCM+. Cavitation patterns show good agreement with experimental measurements, especially the blade tip refined meshes which captured the dynamic behaviour of tip vortex cavitation. The predicted pressure pulse amplitudes agree reasonably well with experimental measurements up to $3^{\text {rd }}$ to $4^{\text {th }}$ order of blade passing frequency.
\end{abstract}

Keywords: propeller; cavitation; hull pressure fluctuation; tip vortex cavitation; RANS

\section{Introduction}

The demand for the prediction of pressure pulses from operating marine propellers is increasing, due to concern for environmental impact and the comfort of onboard passengers and crew. The quest for higher efficiency also calls for more accurate estimations of the pressure pulses, as there is often a trade-off between the two. Numerical prediction of pressure pulses is a challenging task because of the complexity of involved physical phenomenon, including the interaction between the ship wake and propeller, different kinds of cavitation occurring on the propeller and in the tip vortex. The Blade Passing Frequency (BPF) is usually used to analyse the propeller induced pressure pulses, and it is believed that the $1^{\text {st }}$ and $2^{\text {nd }}$ order BPF fluctuations are caused by the blade load and growth and shrinkage of propeller sheet cavitation while tip vortex cavitation is the source of higher order pressure fluctuations, even though the mechanisms are still unclear. In the present study, a model scale container vessel with a five bladed propeller was simulated and results are compared with experiments performed by HSVA in the VIRTUE and SONIC EU projects.

Successful predictions of the sheet cavitation extent and related $1^{\text {st }}$ order pressure fluctuations have been reported [1] [2]. Regarding prediction of the tip vortex, its strength, at least the minimum pressure, could be predicted well close to the propeller tip [3] [4]. Using scale-resolved RANS models with tip mesh refinement, also tip vortex cavitation has been predicted [5] [6]. In the present study, the commercial package Star-CCM+ was used to solve the incompressible flow with the $k-\omega S S T$ turbulence model and the Schnerr-Sauer mass transfer model. Different meshes have been tested where the finest mesh consists of about 30 million cells, including a propeller blade tip refinement region used to capture the tip vortex and its interaction with the sheet cavitation.

\section{Simulation set up}

The studied container vessel was developed in 2002 with bulbous bow and single screw five bladed fixed pitch propeller of diameter $D=7.9 \mathrm{~m}$ with tip clearance of $0.277 D$. The model scale ship (scale ratio 1:29.1) was tested in the cavitation tunnel HYKAT at HSVA. It was installed according to full scale draft of $11.3 \mathrm{~m}$ while the free surface was substituted by flat plates. 13 probes were placed on the hull body to measure the propeller induced hull pressure fluctuations. The arrangement of probe locations is shown in figure 1. Two operating model conditions have been considered in the present study, with same thrust coefficient $K_{T}=\frac{T}{\rho n^{2} D^{2}}$ and cavitation number $\sigma_{n}=\frac{p-p_{v}}{0.5 \rho(\pi n D)^{2}}$ but based on different approaches of scaling correspond to towing tank condition and cavitation tunnel condition, as shown in table 1 . Both conditions correspond to full scale ship cruising speed of 23.76 knots.

\begin{tabular}{lcccc} 
Case & Inlet velocity & Rotation rate & Thrust coefficient & Cavitation number \\
\hline Condition 1 & $2.216 \mathrm{~m} / \mathrm{s}$ & $8.5 \mathrm{rps}$ & 0.2234 & 0.2354 \\
Condition 2 & $7.268 \mathrm{~m} / \mathrm{s}$ & $28 \mathrm{rps}$ & 0.2234 & 0.2354
\end{tabular}

Table 1: Simulation conditions

\footnotetext{
*Muye Ge, muye.ge@chalmers.se
} 

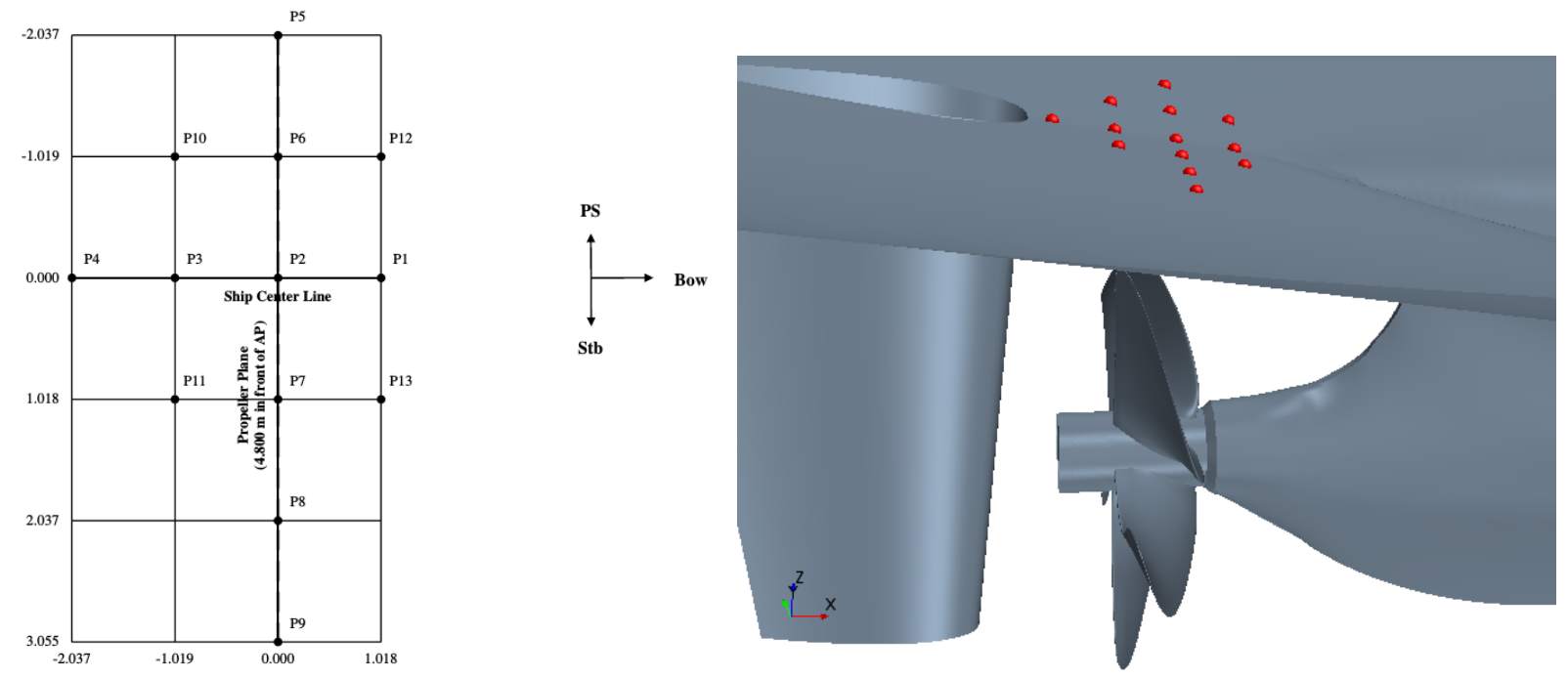

Figure 1: Pressure sensors configuration during model test

\section{Mesh}

The simulation domain corrsponds with the model test tunnel section, as shown in figure 2. The meshes were generated using Pointwise. Structured mesh was applied on the blades (31 layers) and hull body surfaces (34 layers) and hybrid extrusion method was used to create the boundary layer prisms. Tetrahedral cells were used to fill the domain volume. A cylinder region was created for the propeller rotation and connected with the outer region by a sliding interface. The propeller blades tip regions were further refined by structured meshed helix-like regions (core size $0.086 \mathrm{~mm}$ ) to have a better capture of the tip vortex behaviour. The target $y^{+}$value is 1 on both propeller blades and hull body.

\begin{tabular}{lccccccc}
$\#$ cells $/ 10^{6}$ & Tets & Pyramids & Prisms & Hexes & Total cells & Inner region & Outer region \\
\hline Condition 1 with refinement & 19.46 & 0.47 & 0.11 & 12.66 & 32.69 & 19.77 & 12.92 \\
Condition 1 without refinement & 16.05 & 0.38 & 0.11 & 11.03 & 27.57 & 14.65 & 12.92 \\
Condition 2 with refinement & 18.29 & 0.46 & 0.11 & 13.87 & 32.73 & 21.57 & 11.16 \\
Condition 2 without refinement & 14.66 & 0.38 & 0.11 & 12.24 & 27.38 & 16.22 & 11.16
\end{tabular}

Table 2: Summary of mesh type counts

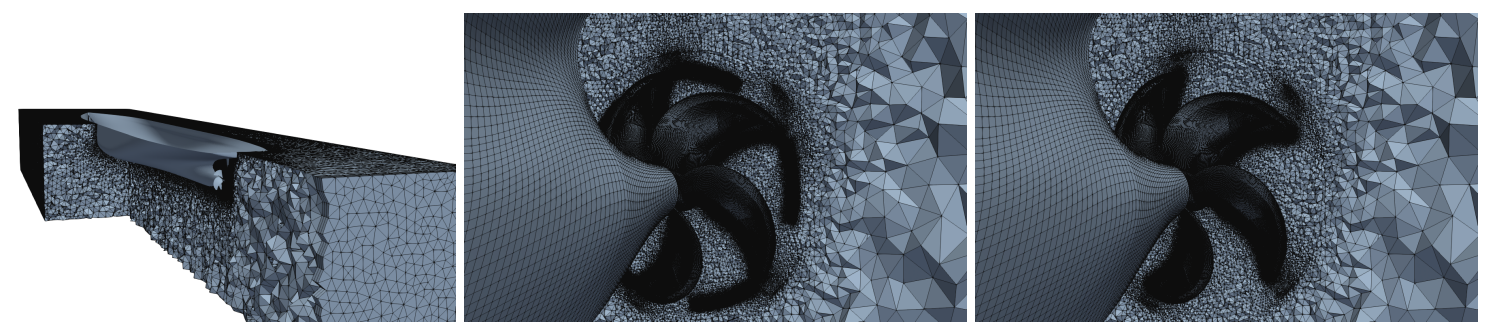

Figure 2: Simulation domain and mesh close to the propeller region

\section{Results}

The major difference between the two studied conditions is the Reynolds number, which would influence the boundary layer and thus the wake where the propeller is operating in. The inlet flow velocity was adjusted to produce same thrust coefficients with the experiment by performing transient non-cavitating simulations. Predicted wakes are shown in figure $3 \mathrm{a}$ and figure $3 \mathrm{~b}$. As shown in the figures, condition 2 has a more compressed wake field than condition 1, but qualitatively similar. The predicted thrust coefficients $K_{T}$ are shown in figure $3 \mathrm{c}$. 


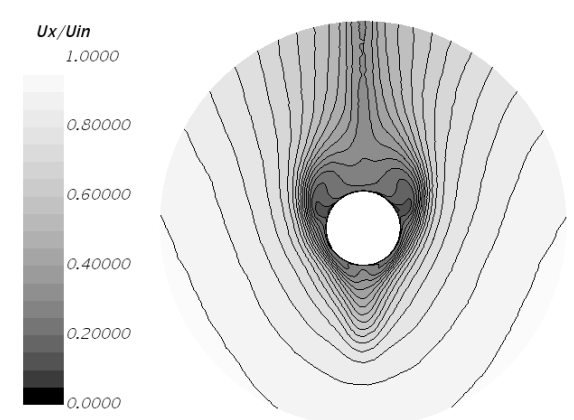

(a) Nominal wake,condition 1

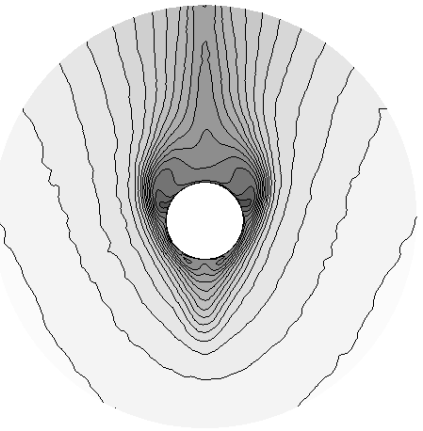

(b) Nominal wake, condition 2

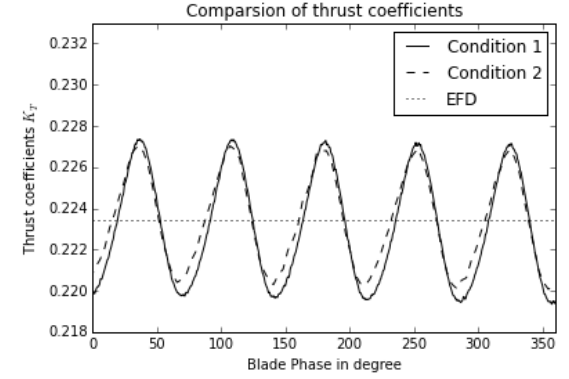

(c) Predicted $K_{T}$ in 1 revolution

Figure 3: Predicted wake and thrust coefficients for non-cavitating cases

For the cavitating conditions, the time step was set to equivalent to 2048 steps per revolution, which is about 5.69 steps per 1 degree revolution. The predicted cavity extent from the tip-refined mesh in condition 1 is shown in figure 4 . During the experiment, the cavitation pattern did not repeat totally from one revolution to the next, and the variation was quite significant. The sketches from experiment demonstrate the stable cavitations in cross lines, which is largely consistent with numerical predictions.

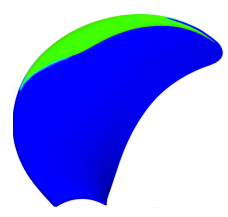

$\phi=180^{\circ}$

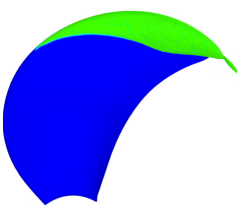

$\phi=210^{\circ}$

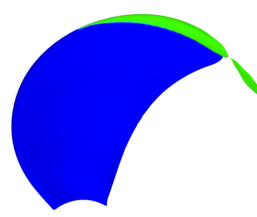

$\phi=240^{\circ}$

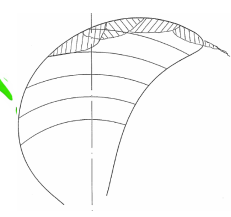

$\phi=180^{\circ}$

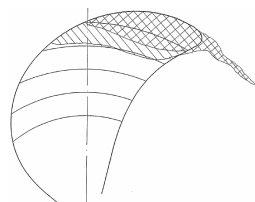

$\phi=210^{\circ}$

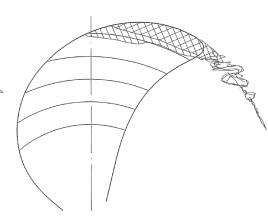

$\phi=240^{\circ}$

Figure 4: Predicted iso-surfaces of $\alpha=0.5$ in condition 1 and cavity sketches from experiment

The predicted tip vortex cavitation show a very unstable behaviour. In figure 5 the cavity is shown close to the blade tip region. The major tip vortex cavitation show a rolling-up structure in a short distance after the blade tip. Unlike the noncavitating case in which only one major tip vortex is predicted close to the blade tip, some secondary vortex structures are formed and rolling with the major tip vortex cavitation, and these secondary vortex would be cavitating in certain conditions.

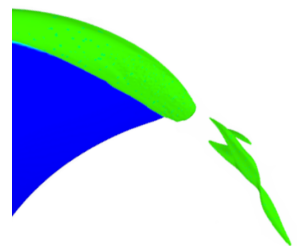

Figure 5: $\phi=232^{\circ}$, tip vortex cavitation

The pressure at each probe and total vapor volume $V_{t, v a p o r}=\Sigma \alpha_{t, i} V_{t, i}$ was recorded during the simulation, as shown in figure 6. Compared to non-cavitating conditions, the pressure fluctuations increased significantly due to the dynamics of vapor structures. The recorded pressure fluctuations follow the same trend as $p \sim d^{2} V_{\text {vapor }} / d t^{2}$. The prediction of tip vortex cavitation increase the total vapor volume and induce more pressure fluctuations.
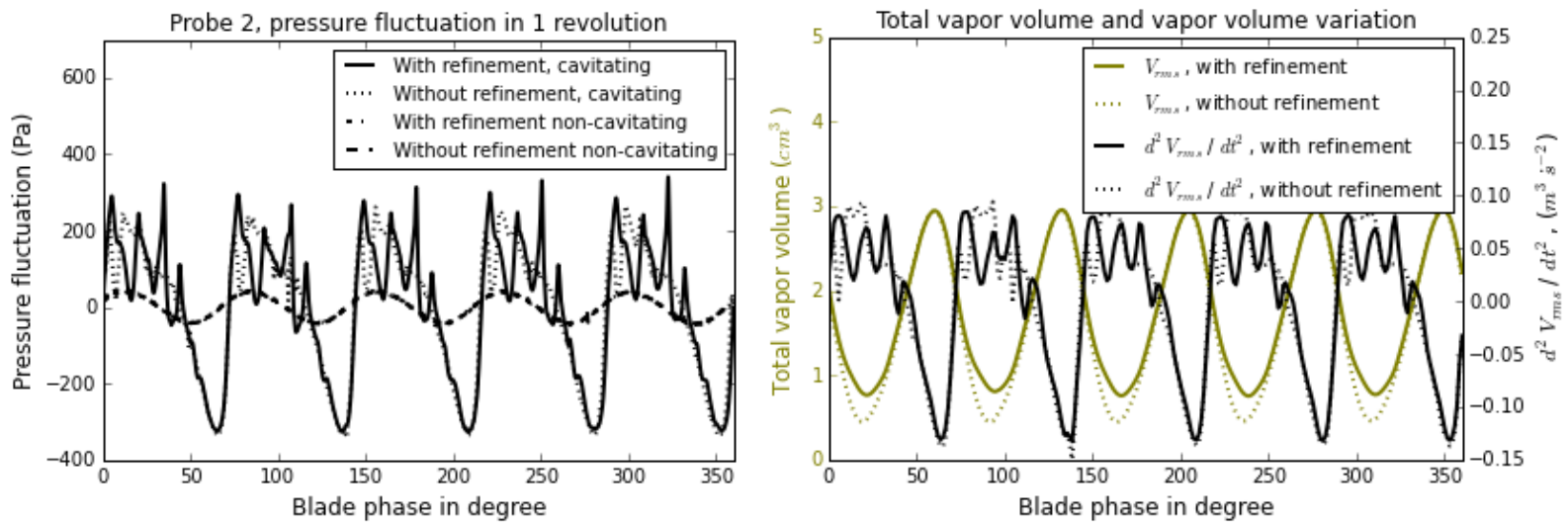

Figure 6: Recorded pressure fluctuations and rms values of total vapor volume in condition 1

The predicted hull pressure amplitudes equivalent to full scale are shown in figure 7 . The results from tip refinement 
meshes predicted lower amplitudes than meshes without refinement for $1^{\text {st }}$ order pressure pulses, slightly higher amplitudes for $2^{\text {nd }}$ order pressure pulses and significantly higher and better results for $3^{\text {rd }}$ order pressure pulses. This is more or less expected, since part of sheet cavity would be rolled up to the tip vortex cavitation and results in a stronger higher order blade passing frequency pulses. Regarding the $4^{\text {th }}$ order pressure pulse amplitudes, rather noticeable values were predicted, but further study is needed to determine if it is physical or originates from numerical errors. The predicted values from condition 2 are overall lower than condition 1 and experimental measurements. One possible explanation is that for condition 2, the residence time of each blade operating in the wake is much shorter than in condition 1 , and the recorded total vapor volume is about $70 \%$ compared to condition 1.
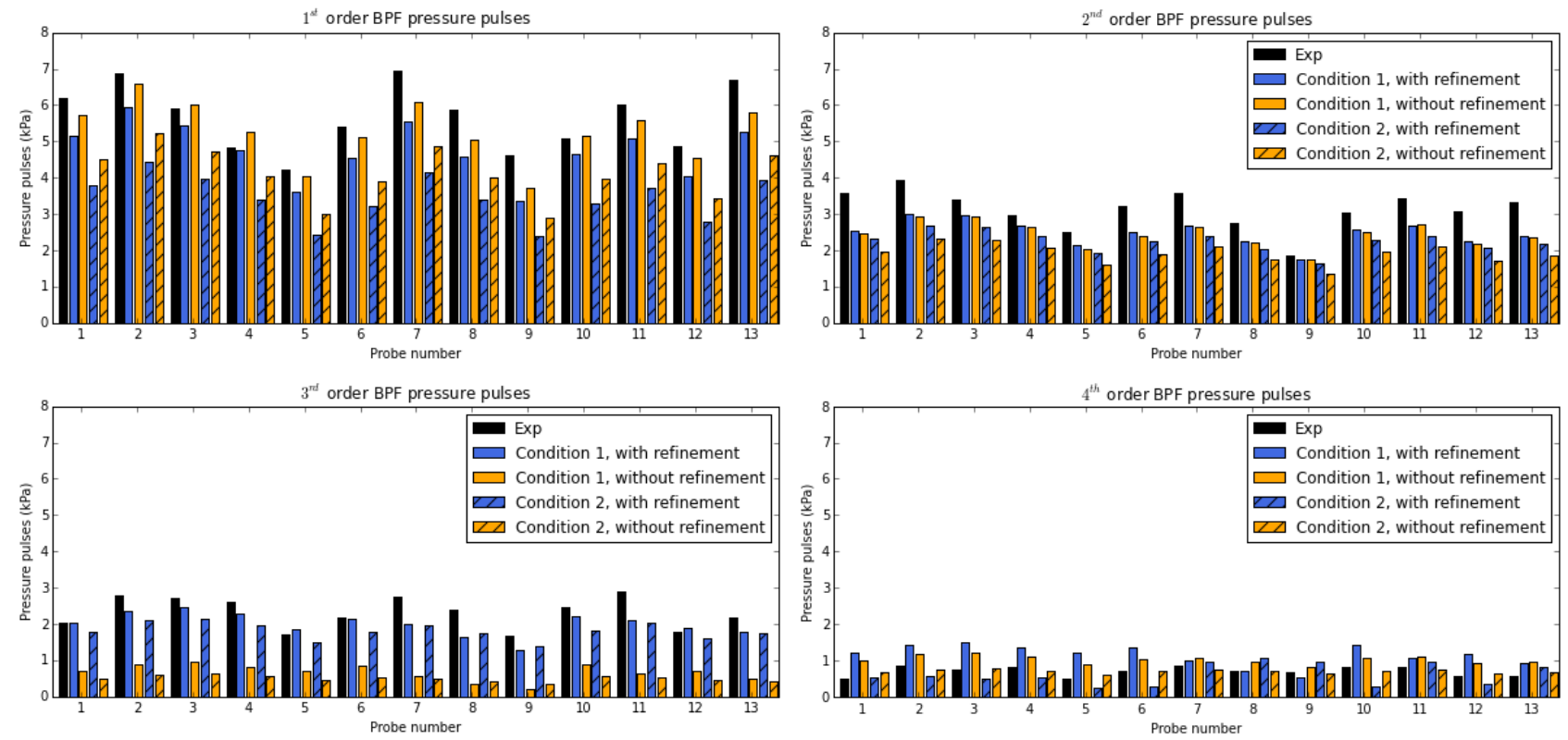

Figure 7: Predicted and measured pressure pulses amplitudes

\section{Conclusion}

Numerical studies for the prediction of pressure pulses induced by an operating marine propeller in behind conditions were presented. Regions of tip refinement were applied close to the propeller tip regions which give better predictions of the dynamic behaviour of tip vortex cavitation. The predicted pressure pulse amplitudes agreed well with experimental data for the simulation condition 1 , while about $70 \%$ of the amplitudes were predicted for the simulation condition 2 . The simulation results also demonstrate the higher order BPF pressure pulses are highly related to the tip vortex cavitation behaviour, and for this case it is possible to be captured by using RANS method.

\section{Acknowledgements}

Financial support for this work has been provided by Rolls-Royce Marine through the University Technology Centre in Computational Hydrodynamics hosted at the Department of Mechanics and Maritime Sciences at Chalmers University of Technology. The computations were performed on resources at Chalmers Centre for Computational Science and Engineering (C3SE) provided by the Swedish National Infrastructure for Computing (SNIC).

\section{References}

[1] K.-J. Paik, H.-G. Park, and J. Seo, "Rans simulation of cavitation and hull pressure fluctuation for marine propeller operating behind-hull condition," International Journal of Naval Architecture and Ocean Engineering, vol. 5, no. 4, pp. 502-512, 2013.

[2] P. Perali, T. Lloyd, and G. Vaz, "Comparison of urans and bem-bem for propeller pressure pulse prediction: E779a propeller in a cavitation tunnel," in Proceedings of 19th Numerical Towing Tank Symposium, 3rd-4th October, St. Pierre dOléron, France, 2016.

[3] C. Hsiao and G. Chahine, "Scaling of tip vortex cavitation inception for a marine open propeller," in 27th Symposium on Naval Hydrodynamics, Seoul, Korea, 2008, pp. 5-10.

[4] A. Asnaghi, R. E. Bensow, and U. Svennberg, "Comparative analysis of tip vortex flow using rans and les," 2017.

[5] K. Fujiyama, "Numerical simulation of ship hull pressure fluctuation induced by cavitation on propeller with capturing the tip vortex," in Fourth International Symposium on Marine Propulsors, 2015.

[6] S. Gaggero, G. Tani, M. Viviani, and F. Conti, "A study on the numerical prediction of propellers cavitating tip vortex," Ocean Engineering, vol. 92, pp. 137-161, 2014. 